\title{
Screening ssDNA aptamers against HIV P24 antigen using agarose beads as carriers
}

\author{
Jiayu Zeng ${ }^{1}$, Xing $\mathrm{Li}^{1}$, Hongxia Yuan ${ }^{2}$, Meilan $\mathrm{Ma}^{1}$, Dongdong $\mathrm{Li}^{2}$, Jing $\mathrm{Ma}^{2}$ and Shiqi Liao ${ }^{2, a}$ \\ ${ }^{1}$ College of Life Sciences, Northwest Normal University, Lanzhou, 730070, China \\ ${ }^{2}$ Laboratory of Molecular Biology, Gansu Academy of Medical Sciences, Lanzhou, 730050, China
}

\begin{abstract}
In this study, carboxylated agarose beads were used as screening carriers combined with the subtractive systematic evolution of ligands by exponential enrichment (SELEX) technology to obtain an ssDNA secondary library, which could specifically bind to HIV P24 antigen after nine screening rounds from a random ssDNA library. An electrophoretic mobility shift assay (EMSA) and quantitative real-time PCR assay (RT-qPCR) was performed to monitor in real-time the binding specificity of the ssDNA secondary library to the HIV P24 antigen. The obtained ssDNA secondary library was converted to the corresponding dsDNA library, ligated to a PMD18-T vector, and transformed into E. coli DH5 $\alpha$ and sequenced. The results demonstrated that after nine rounds of screening, two aptamer sequences specifically bound to the HIV P24 antigen were obtained.
\end{abstract}

\section{Introduction}

Currently, the diagnosis and treatment of human AIDS virus (HIV)-caused acquired immune deficiency syndrome (AIDS) is still a medical issue. Early diagnosis of AIDS is an important part of treatment. Predicting the spread possibility of AIDS and evaluating treatment effects both require highly sensitive and specific diagnostic methods. There are many ways to measure HIV infection; detecting the HIV P24 antigen and its antibody is one of the most commonly used methods in HIV clinical screenings.

The HIV P24 antigen is a major structural component of the HIV envelope, with a molecular weight of $24 \mathrm{KD}$. Each complete virus particle contains approximately 2,000 HIV P24 antigen molecules. During early HIV infection, the HIV P24 antigen expresses in the human body and can be detected in the blood. However, the induced HIV P24 antibody subsequently binds to the HIV P24 antigen, which makes it difficult to detect the HIV P24 antigen in the blood.

Systematic evolution of ligands by exponential enrichment technique (SELEX) is a novel in vitro screening technique that has emerged in the last 20 years. It combines library technology and screening technology to screen out ssDNA oligonucleotide molecules, the so-called aptamers from a random ssDNA library. Aptamers are similar to antibodies because they can bind to the corresponding targets with high affinity and specificity. Currently, screening aptamers with SELEX is developing quickly. Aptamers can be widely applied in various fields, including medical biology. In terms of virus research and treatment, SELEX has been used to screen out various relevant aptamers of viruses,

\footnotetext{
${ }^{a}$ Corresponding author
}

This work was supported by Chinese National Science Foundation (81360333; 81560346). 
providing a new direction for preventing and treating related diseases.

In this study, SELEX was applied to screen aptamers for the HIV P24 antigen, laying the foundation for new methods of AIDS diagnosis and treatment.

\section{Materials and Methods}

\subsection{Materials}

Carboxylated agarose beads were provided by Gansu Academy of Medical Sciences; the HIV P24 antigen $(2 \mathrm{mg} / \mathrm{ml})$ was from Beijing Wanyumeilan Reagent Company; Streptavidin was synthesized by TaKaRa; the PMD18-T vector system was from Shanghai Sangon Biotech Co., Ltd.; the E. coli DH5 $\alpha$ was from Gansu Academy of Medical Sciences; the random ssDNA library was synthesized by Shanghai Sangon Biotech Co., Ltd. with the following sequence: 5'CTATAGCAATGGTACGGGTAC-TTCC-(40N)-CAAAAGTGCACGCTACTTTGCTAA-3' (N = A, G, T, C); primer P7 with the sequence 5'-CTATAGCAATGGTACGGTACT-TCC-3' and primer P11 with the sequence 5'-TTAGCA-AAGTAGCGTGG-ACTTTTG-3' was synthesized by TaKaRa; the anti- HbsAg was from Beijing Wanyumeilan Reagent Company; the PCR-related reagents were from Shanghai Sangon Biotech Co., Ltd. and TaKaRa; all other reagents were analytical grade.

The GeneAmp PCR System 2700PCR instrument was from AppliedBiosystems (USA); The MVT-28 gel imager was from Herolab (Germany); the ROTOR-GENE 3000 Real Time PCR System was from Rotor-Gene (USA); the mini double vertical electrophoresis tank DYCZ-24DN was from Beijing 61 Instrument Factory; the SHZ-82 thermostat shaker was from Changzhou Guohua instruments Co., Ltd.; the TGL-16A high-speed refrigerated centrifuge was from Changsha Pingfan Instruments Co., Ltd.

\subsection{Methods}

\subsubsection{Screening aptamers for the HIV P24 antigen}

\section{1) Screening Round 1}

Coating: The carboxylated agarose beads were placed in a $5 \mathrm{ml}$ Eppendorf tube and washed with $5 \times$ beads volume deionized water three times. A volume of $500 \mu 1100$-fold diluted HIV P24 antigen was added and incubated at $37^{\circ} \mathrm{C}$ for $1 \mathrm{~h}$.

Blocking: The supernatant was discarded and the precipitant was washed three times with 0.01 mol/L pH7.4 PBS. Then, a $1 \mathrm{ml}$ blocking solution $(0.1 \mathrm{~g}$ sucrose, $12.5 \mathrm{mg}$ bovine serum albumin, and $12.5 \mathrm{mg}$ casein dissolved in $10 \mathrm{ml}$ PBS) was added and incubated at $37{ }^{\circ} \mathrm{C}$ for $1 \mathrm{~h}$. Again, the supernatant was discarded and the precipitant was rinsed with $0.01 \mathrm{~mol} / \mathrm{L} \mathrm{pH} 7.4 \mathrm{PBS}$ three times. Then, it was transferred to a new Eppendorf tube.

Pretreatment of the ssDNA library: The new synthesized random ssDNA library was dissolved in a $300 \mathrm{ml}$ binding buffer, heated in a boiling water bath for $5 \mathrm{~min}$, and was then rapidly cooled for 10 min.

Binding: The pretreated ssDNA library was added to the abovementioned Eppendorf tube and incubated at $37{ }^{\circ} \mathrm{C}$ for $2 \mathrm{~h}$. The supernatant was discarded and the precipitant was washed with a $2 \times$ saline-sodium citrate (SSC) solution 5 times, and rinsed with pure water after replacing the tube.

Eluting: The supernatant was discarded. Then, $100 \mu 1$ of water was added to the Eppendorf tube and boiled in a boiling water bath for $5 \mathrm{~min}$. After it was centrifuged at $12,000 \mathrm{rmp}$ for $5 \mathrm{~min}$, the supernatant was collected. This step was repeated three times and the supernatants were combined.

Preparation of the dsDNA library [12]: A sample of $80 \mu 1$ of recovered supernatant was used as a template to prepare dsDNA in a $100 \mu \mathrm{PCR}$ reaction. Besides the template, the PCR solution included a $10 \mu 1$ 10×Buffer, fluorescent dye supergreen I $1.5 \mu 1$, dNTP mixture $(25 \mathrm{mmol} / \mathrm{L}$ of each dNTP) 0.8 $\mu 1$, primer P7 $1.0 \mu$, 5'-biotinylated primer P11 $1.0 \mu$, and TapDNA polymerase $0.5 \mu 1$, added with 
deionized water to a total volume of $100 \mu 1$. The $100 \mu$ l solution was aliquoted into PCR tubes with 24 $\mu 1$ per tube to perform PCR amplification at the following conditions: $95{ }^{\circ} \mathrm{C}$ for $6 \mathrm{~s}$, then $60{ }^{\circ} \mathrm{C}$ for 34 $\mathrm{s}$; after reaching the S-curve peak, the PCR amplification was terminated. Thus, the biotinylated dsDNA was obtained.

Preparation of the ssDNA library [12]: The prepared streptavidin-biotin magnetic beads were rinsed three times in $0.01 \mathrm{~mol} / \mathrm{L} \mathrm{pH7.4} \mathrm{PBS}$. The biotin-labeled dsDNA from the last step was added to the beads and incubated in a rotating shaker for $30 \mathrm{~min}$ at $37{ }^{\circ} \mathrm{C}$. The supernatant was discarded, and then a sample of $0.4 \mathrm{ml} \mathrm{5 \%}$ formamide was added and incubated in a $40{ }^{\circ} \mathrm{C}$ water bath for $3 \mathrm{~min}$. After it was rinsed 4 times with deionized water, the sample was transferred into a new Eppendorf tube. A sample of a $200 \mu \mathrm{l}$ binding buffer was added into the tube and incubated in a $100{ }^{\circ} \mathrm{C}$ dry block heater for $5 \mathrm{~min}$. The Eppendorf tube was removed and the supernatant was transferred into a new tube. Thus, the ssDNA secondary library was obtained and to be used for the next round of screening.

\section{2) Screening Round 2}

Coating: The carboxylated agarose beads were washed with deionized water with $5 \times$ beads volume deionized water three times and evenly allocated into two Eppendorf tubes, labelled as the positive and negative selection tube. In the positive selection tube, a volume of $500 \mu 1100$-fold diluted HIV P24 antigen was added; in the negative tube, the same volume of 50-fold diluted Anti-HBsAg was added, and both tubes were incubated at $37^{\circ} \mathrm{C}$ for $1 \mathrm{~h}$.

Blocking: For both of the positive and negative selection tubes, the supernatants were discarded and the precipitants were washed three times with $0.01 \mathrm{~mol} / \mathrm{L} \mathrm{pH7.4} \mathrm{PBS.} \mathrm{Then,} \mathrm{a} \mathrm{sample} \mathrm{of} \mathrm{a} 1 \mathrm{ml}$ blocking solution was added into each tube and incubated in a $37{ }^{\circ} \mathrm{C}$ rotating shaker for $1 \mathrm{~h}$.

Binding: The supernatants in both tubes were discarded and the precipitants were each washed with $0.01 \mathrm{~mol} / \mathrm{L} \mathrm{pH7.4} \mathrm{PBS} \mathrm{three} \mathrm{times.} \mathrm{The} \mathrm{ssDNA} \mathrm{secondary} \mathrm{library} \mathrm{obtained} \mathrm{from} \mathrm{the} \mathrm{last} \mathrm{round}$ was added to the negative selection tube and incubated at $37^{\circ} \mathrm{C}$ for $2 \mathrm{~h}$. Then, the supernatant from this tube was transferred into the positive selection tube and incubated at $37{ }^{\circ} \mathrm{C}$ for another $2 \mathrm{~h}$. The supernatant from the positive selection tube was discarded. Then, the precipitant from each tube was washed five times with a $2 \times \mathrm{SSC}$ solution and rinsed with pure water after replacing the tubes.

Eluting: A sample of $100 \mu \mathrm{l}$ pure water was added to each tube and boiled in a boiling water bath for $5 \mathrm{~min}$. After it was centrifuged at $12,000 \mathrm{rmp}$ for $5 \mathrm{~min}$, the supernatant from each tube was collected. This step was repeated three times and the supernatant was combined from each tube, respectively.

The preparation of the ssDNA secondary library was the same as in the first round of screening.

\section{3) Re-screening}

In subsequent screening process rounds, the HIV P24 antigen was always used as the target protein for the positive selection, and the anti-HbsAg and goat polyclonal HIV-P24 antibody were used alternatively as the non-target protein for the negative selection. In each screening round, the obtained ssDNA secondary library was used as the starting library for the next round, whereas all other operations were the same as the second round. Meanwhile, in each round, RT-qPCR was performed to monitor the enrichment of target ssDNA until it reached the maximum. This completed the screening process.

\section{4) Determining the ssDNA library enrichment}

RT-qPCR was used to determine the ssDNA library enrichment. The PCR solution included the ssDNA library $6.0 \mu \mathrm{l}$ as a template, $10 \times$ Buffer $10 \mu \mathrm{l}$, dNTP mixture $(25 \mathrm{mmol} / \mathrm{L}$ of each dNTP) $0.8 \mu 1$, primer P7 $0.4 \mu 1,5$ '-biotinylated primer P11 $0.4 \mu$, TapDNA polymerase $2.0 \mu 1$, and fluorescent dye $1.0 \mu \mathrm{l}$, with added deionized water to a total volume of $100 \mu \mathrm{l}$. After it was rapidly and thoroughly mixed, the solution was aliquoted into PCR tubes at $24 \mu 1$ per tube. The PCR reaction condition was $95{ }^{\circ} \mathrm{C}$ for $6 \mathrm{~s}$ and $60{ }^{\circ} \mathrm{C}$ for $4 \mathrm{~s}$, for a total of 40 cycles. The negative control was prepared with deionized water as a template, and the positive one was prepared with the ssDNA starting library as a template, with all other ingredients and steps the same as above. 


\subsubsection{Determining the specific binding of ssDNA with the target protein}

1) The electrophoretic mobility shift assay (EMSA) was performed to determine the specificity of HIV P24 antigen-ssDNA complexes formed by ssDNA and the target protein. A sample of $5 \mu 1 \mathrm{HIV}$ P24 antigen was mixed with $10 \mu \mathrm{l}$ ssDNA and incubated at $37^{\circ} \mathrm{C}$ for $1 \mathrm{~h}$. Then, the agarose gel electrophoresis was conducted with the following condition: $1.5 \% \mathrm{~W} / \mathrm{V}$ agarose, $1 \times \mathrm{TAE}$ buffer, a constant voltage of $80 \mathrm{~V}$ and 40 min electrophoresis. At the same time, two controls, the HIV P24 antigen and ssDNA, were set up and the gel was stained with Coomassie blue.

2) RT-qPCR was conducted to determine the binding specificity between the ssDNA and the target protein, the HIV P24 antigen. Two samples of the same amount of carboxylated agarose beads were coated with the HIV P24 antigen and the non-target protein, Anti-HbsAg, respectively. According to the screening method for round 1 in section 1.2.1, the ssDNA libraries bound with the target protein and the non-target protein were prepared respectively. Afterwards, the prepared ssDNA libraries were used as templates for RT-qPCR according to the method for determining the ssDNA library enrichment in section 1.2.1.

\subsubsection{Sequencing}

The ssDNA aptamers library which specifically bound to the target protein HIV P24 antigen that was used to prepare the corresponding dsDNA library (with the downstream primer of 5'-biotinylated P11 instead and all other operations the same as the screening method for round 1 in section 1.2.1). Then, the dsDNA library was ligated to the PMD TM 18-T vector and transformed into E. coli DH5 $\alpha$. The positive clones were sent for sequencing at the Shanghai Personalbio Biological Technology Co., Ltd. The ligation and transformation followed conventional methods.

\section{Results and Analysis}

\subsection{Screening HIV P24 antigen aptamers}

In the newly synthesized ssDNA library, the abundance of ssDNA specifically bound to the target protein was low. Therefore, the negative selection process was skipped in the first round. The ssDNA enrichments of the first round determined by RT-qPCR demonstrated clearly separated PCR curves for the positive control, the screening sample, and the negative control (Figure 1), with a significantly higher DNA abundance in the screening tube than in the negative control. This indicated that the target protein in the screening tube bound a certain amount of ssDNA. Subsequently, the ssDNA served as a template for preparing the ssDNA secondary library, the starting library for the next round. 


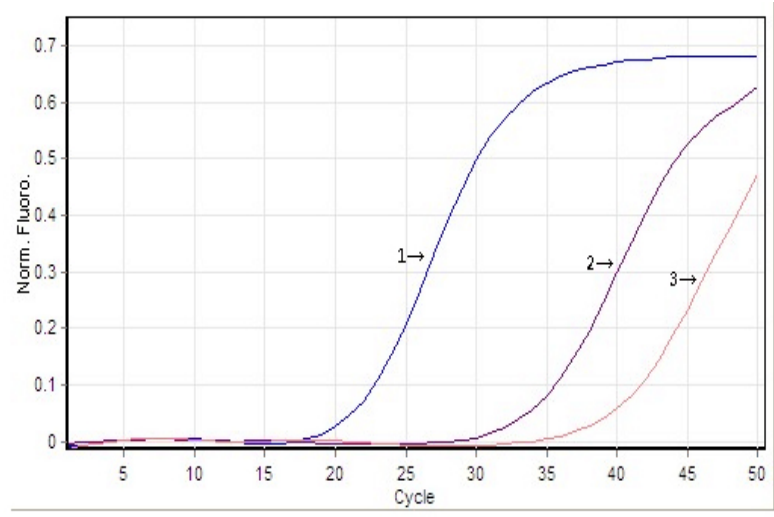

Figure 1. RT-qPCR determination of the ssDNA enrichment for the first round of screening. 1. The positive control; 2. The screening tube; 3 . The negative control. The vertical axis is the fluorescence intensity, and the horizontal axis is the number of PCR cycles. After 18 cycles, the ssDNA in the positive control started to enrich; after 27 cycles, the ssDNA in the screening tube started to enrich; after 33 cycles, the ssDNA in the negative control started to enrich.

After the first round of screening, a large amount of non-target ssDNA was removed and the abundance of the target ssDNA was greatly improved. Hence, to more accurately evaluate the target ssDNA abundance, the negative selection was established from the second round of screening. From the RT-qPCR results for rounds 2-4 of screening, the PCR curves were distinct for the positive selection tube, and the positive and negative controls; the PCR curves were distinct for the negative selection tube, and the positive and negative controls. The PCR curves for the positive and negative selection tubes were not distinct from each other. Figure 2 demonstrates the RT-qPCR results for round 4 of screening. The results indicated that during screening rounds $2-4$, both the positive and negative selections enriched ssDNA. However, the largely enriched ssDNA in the negative selection indicated high abundant non-target ssDNA in the starting libraries, and a low abundance of the target ssDNA.

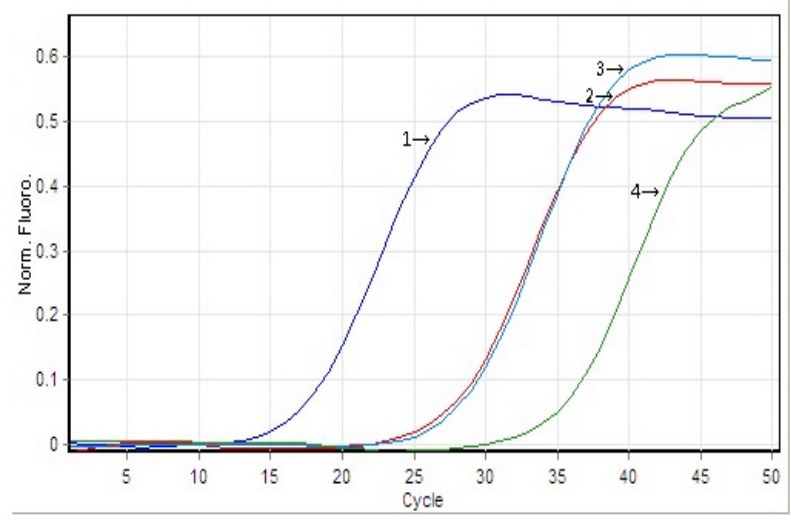

Figure 2. RT-qPCR determination of the ssDNA enrichment for the fourth round of screening. 1. The positive control; 2 . The positive selection tube; 3 . The negative selection tube; 4 . The negative control. The vertical axis is the fluorescence intensity, and the horizontal axis is the number of PCR cycles. After 14 cycles, the ssDNA in the positive control started to enrich; after 23 cycles, the ssDNA in the positive and negative selection started to enrich; after 26 cycles, the ssDNA in the negative control started to enrich.

Using the ssDNA secondary library from round 4 as the starting library, the $5^{\text {th }}$ round of screening was conducted. Figure 3 shows the RT-qPCR results for the $5^{\text {th }}$ round. The results demonstrated 
distinct PCR curves for the positive selection tube, and the positive and negative controls; it also showed distinct PCR curves for the negative selection tube, and the positive and negative controls. Meanwhile, the PCR curves for the positive and negative selection tubes started to separate, indicating that the ssDNA abundance was different between them, with a one cycle difference. This suggested the presence of non-target ssDNA in the starting library of round 5, only with a low abundance. At the same time, the ssDNA that was specifically bound with the HIV P24 antigen started to enrich.

Subsequently, a continuous five rounds of screening was conducted. As shown in the RT-qPCR results for rounds 6-10, the PCR curves for the positive and negative selection tubes separated further and further. The curves for the negative selection became closer to the negative control, and the difference was only one cycle for round 9. There were no significant differences between screening rounds 9 and 10, as shown from the PCR results. These results indicated that during rounds $6-10$, the target ssDNA was enriching, and the non-target ssDNA was disappearing. At round 9, the abundance of the non-target ssDNA reached the lowest point, while the target ssDNA reached the highest point. Therefore, the obtained ssDNA secondary library was the target ssDNA that bound specifically to the target HIV P24 antigen, the so-called ssDNA aptamers for the HIV P24 antigen. Figure 4 shows the RT-qPCR results for the $9^{\text {th }}$ round of screening.

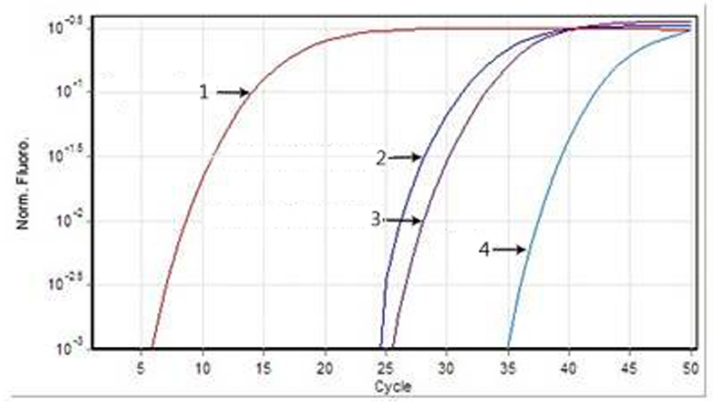

Figure 3. RT-qPCR determination of the ssDNA enrichment for the fifth round of screening. 1. The positive control; 2 . The positive selection tube; 3 . The negative selection tube; 4 . The negative control. The vertical axis is the fluorescence intensity, and the horizontal axis is the number of PCR cycles. After 6 cycles, the ssDNA in the positive control started to enrich; after 25 cycles, the ssDNA in the positive selection started to enrich; after 26 cycles, the ssDNA in the negative selection started to enrich; after 35 cycles, the ssDNA in the negative control started to enrich.

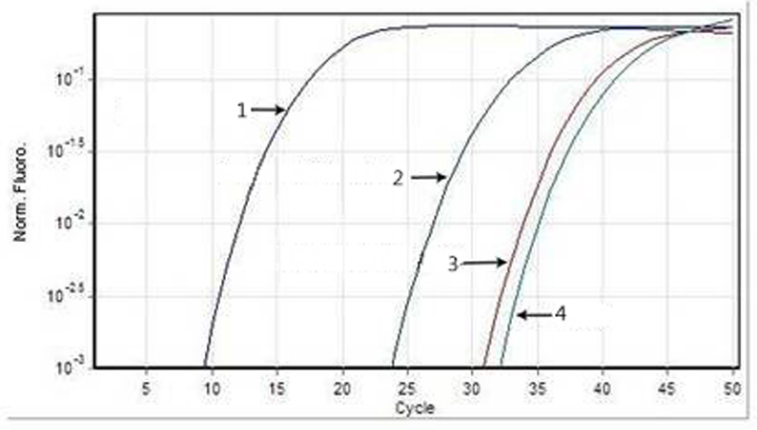

Figure 4. RT-qPCR determination of the ssDNA enrichment for the ninth round of screening. 1. The positive control; 2 . The positive selection tube; 3 . The negative selection tube; 4 . The negative control. The vertical axis is the fluorescence intensity, and the horizontal axis is the number of PCR cycles. After 9 cycles, the ssDNA in the positive control started to enrich; after 24 cycles, the ssDNA in the positive selection started to enrich; after 31 cycles, the ssDNA in the negative selection started to enrich; after 32 cycles, the ssDNA in the negative control started to enrich. 


\subsection{Preparation of the ssDNA secondary Library}

The ssDNA secondary library obtained after the $9^{\text {th }}$ round of screening was the target of ssDNA aptamers that could specifically bind to the HIV P24 antigen. The preparation of the ssDNA secondary library included two steps. First, the supernatant ssDNA product of the $9^{\text {th }}$ round of screening was PCR amplified to prepare the biotinylated dsDNA library. Second, the streptavidinbiotin beads were used to prepare the corresponding ssDNA library from the above biotinylated dsDNA library. Thus the ssDNA aptamers were obtained, and the methods were as described for the first round of screening.

Figure 5 shows the RT-qPCR results of the preparation of the biotinylated dsDNA library. It was demonstrated that after about 15 cycles, the DNA started to enrich, and reached the $\mathrm{S}$ curve peak after 35 cycles for all four parallel PCR tubes. Figure 6 demonstrates the polyacrylamide gel electrophoresis (PAGE) results of the prepared dsDNA and ssDNA libraries. There were clear bands of approximately $80 \mathrm{bp}$ at lanes 1 and 2, consistent with the molecular weight of the synthesized random library, which indicated the acquisition of the dsDNA and ssDNA libraries. In addition, the dsDNA had a higher mobility rate due to the formation of a compact double helix structure.

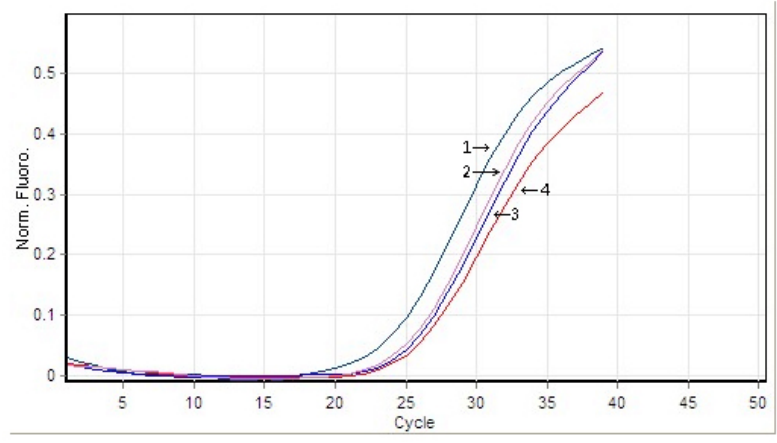

Figure 5. RT-qPCR determination of the preparation of the dsDNA library. 1, 2, 3, and 4 are four parallel PCR tubes, all templated with the ssDNA secondary library from the ninth round of screening. The vertical axis is the fluorescence intensity, and the horizontal axis is the number of PCR cycles.

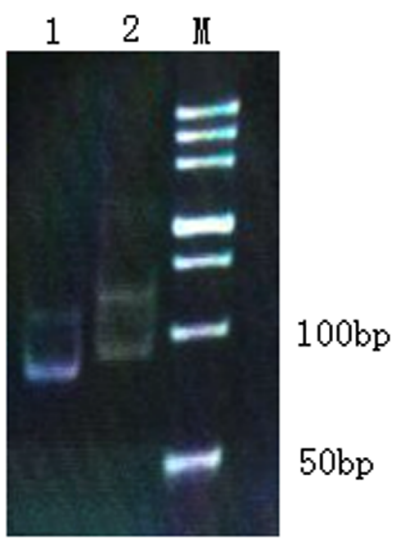

Figure 6. PAGE detection of the prepared dsDNA and ssDNA libraries. Lane M. DNA markers; Lane 1. dsDNA sample; Lane 2. ssDNA sample. A $10 \%$ non-denaturing polyacrylamide gel was used with a loading volume of 1 $\mu \mathrm{L}$.

\subsection{EMSA identification of the aptamers}

The ssDNA library of the aptamers of the $9^{\text {th }}$ round of screening should be able to recognize and bind 
to the target protein HIV P24 antigen specifically to form HIV P24 antigen-ssDNA complexes. The molecular weight and charge properties of the complexes were different from the HIV P24 antigen and ssDNA, causing different electrophoretic mobility rates. Thus, EMSA was conducted to identify the HIV P24 antigen-ssDNA complexes. Figure 7 shows the PAGE results. As shown in this figure, there was a clear band in lanes 1 and 2, with lane 2 showing a higher mobility rate than lane 1 . This result suggested that the HIV P24 antigen bound with ssDNA aptamers and formed HIV P24 antigenssDNA complexes. Since the complexes had higher molecular weights than the HIV P24 antigen, they encountered large gel retardation and resulted in decreased mobility. Lane 3 was ssDNA and would not be stained by Coomassie blue.

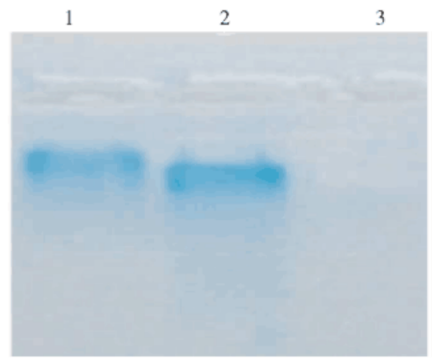

Figure 7. The $1 \%$ agarose gel results of the aptamer complexes identified with EMSA. Lane 1 . HIV P24 antigenssDNA complexes; Lane 2. HIV P24 antigen; Lane 3. ssDNA. A 10\% non-denaturing polyacrylamide gel was used with a loading volume of $10 \mu \mathrm{l}$.

\subsection{Identification of binding specificity of SSDNA and HIV P24 antigen by RT-qPCR}

The ssDNA library of the aptamers obtained from the $9^{\text {th }}$ round of screening was bound to the beads coated with the target protein HIV P24 antigen and the non-target protein Anti-HbsAg, respectively, to prepare the specific binding ssDNA secondary libraries. RT-qPCR was performed to monitor the ssDNA abundance in the secondary libraries; Figure 8 shows the results.

The results showed distinct enrichment curves for the HIV P24 antigen-coated tube and the negative control, indicating a high abundance of ssDNA in the HIV P24 antigen-coated tube. Which meant that the beads-coated with the HIV P24 antigen captured large amounts of ssDNA. The enrichment curves for the Anti-HbsAg-coated tube and the negative control nearly superimposed each other, indicating little to no ssDNA in the Anti-HbsAg-coated tube. This meant that the beads-coated with Anti-HbsAg failed to capture ssDNA. The above results further confirmed that the ssDNA obtained from the $9^{\text {th }}$ round of screening could specifically bind to the HIV P24 antigen, and it could serve as HIV P24 antigen aptamers.

Moreover, from the experimental results, it can be seen that the enrichment curves for the AntiHbsAg-coated tube and the negative control were not identical. This might be due to the non-specific binding between ssDNA and Anti-HbsAg, or an incomplete wash. 


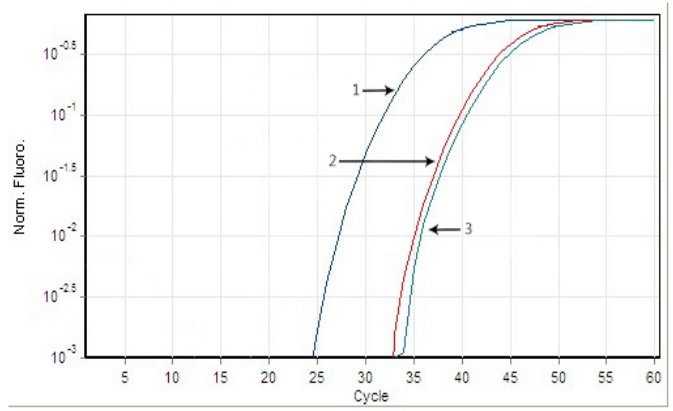

Figure 8. The binding specificity between ssDNA and HIV P24 antigen identified by RT-qPCR. 1. HIV P24 antigen-coated tube; 2. Anti-HbsAg-coated tube; 3 . The negative control. The vertical axis is the fluorescence intensity, and the horizontal axis is the number of PCR cycles. After 25 cycles, the ssDNA in the HIV P24 antigen-coated tube started to enrich; after 33 cycles the ssDNA in the Anti-HbsAg-coated tube started to enrich; after 34 cycles, the ssDNA in the negative control started to enrich.

\subsection{Sequencing}

The obtained ssDNA library of aptamers for HIV P24 antigen was converted into a dsDNA library and transformed into E. coli DH5a. Three positive clones were picked and sent for sequencing and chemical synthesis at Shanghai Personalbio Biological Technology Co., Ltd. The three synthesized ssDNA were amplified by PCR and the HIV P24 antigen binding specificity was confirmed by EMSA and RT-qPCR. Eventually, two HIV P24 antigen-specific binding aptamers were obtained, with sequences as follows:

Chain 1:

\section{5'-TCGGCACGTTCTCAGTAGCGCTCGCTGGTCATCCCACAGCTACGT-3';}

Chain 2:

\section{5’-GGACGCGGTTAGGGGAGAATTTCCATACCTTTGGGTTGTA-3’'}

\section{Discussion}

Due to its unique advantages, aptamer plays a very important role in molecular diagnostics, molecular imaging, targeting delivery, and treatment of cancer. Aptamers have structural diversity, demonstrating a high specificity and a strong affinity upon binding to a variety of target proteins. In terms of protein detection, aptamers are superior to the conventional antigen-antibody means [11-12], and has great potential for broad applications.

This study combined subtractive SELEX and RT-qPCR, using carboxylated agarose beads as the carrier and HIV-P24 antigen as the target molecule, to screen specific oligonucleotide aptamers. Compared to conventional screening techniques, this method is inexpensive, simple, fast, and can maximize the target ssDNA enrichment with high screening efficiency and specificity. In addition, the use of carboxylated agarose beads could largely avoid contamination and hard separation caused by other traditional carriers. Carboxylated agarose beads could improve the coupling efficiency between the target protein and the carrier. The streptavidin-biotin magnetic beads would improve the preparation efficiency and the purity of ssDNA because of the coupling effect for dsDNA and the carrier due to high affinity between streptavidin and biotin. Our next step would be to further explore and establish new approaches for screening and preparing ssDNA aptamers. Improving the screening efficiency would be an important part of our future work.

After 9 rounds of screening, two aptamer sequences specifically binding to HIV P24 antigens were obtained. Our study lays the foundation for new approaches to quickly, accurately, and efficiently 
detect HIV P24 antigens with aptamers.

\section{References}

1. Cai D, Xu D, Zhang Q, et al. Classification of lung cancer using ensemble-based feature selection and machine learning methods [J]. Molecular biolystems, 2014, 464(7291): 1071-1076.

2. ZENG Jia-yu, YANG Nan, WAN Bo, et al. The preparation and characterization of epoxymodified magnetic microsphere [J]. Journal of Northwest Normal University (Natural Science), 2014, 50(2): 93-99.

3. Kulbachinskiy AV. Methods for selection of aptamers to protein targets [J]. Biochemistry, 2007, 72: $1505-1518$.

4. Ireson CR, Kelland LR. Discovery and development of anti-cancer aptamers [J]. Mol Cancer Ther, 2006, 5: 2957-2962.

5. Barbas AS, Mi J, Clary BM, et al. Aptamer applications for targeted cancer therapy [J]. Future Oncol, 2010, 6: 1117- 1126.

6. Elyse D. Bernard, Kathy C. Nguyen, Maria C. DeRosa, Azam F. Tayabali, Rocio Aranda Rodriguez. Development of a bead-based aptamer/antibody detection system for Creactive protein. Analytical Biochemistry. 2015: 67-74.

7. Fang X, Tan W. Aptamers generated from cell-SELEX for molecular medicine: a chemical biology approach [J]. Acc Chem Res, 2010, 43: 48-57.

8. Sturgeon JP, Shawcross DL. Recent insights into the pathogenesis of hepatic encephalopathy and treatments [J]. Expert Rev Gastroenterol Hepatol, 2014, 8(1): 83-100.

9. Sefah K, Meng L, Lopez-Colon D, et al. DNA aptamers as molecular probes for colorectal cancer study [J]. PLoS One, 2010, 5: e14269. doi: 10.1371/journal. pone. 0014269.

10. Keefe AD, Pai S, Ellington AD. Aptamers as therapeutics [J]. Nat Rev Drug Discovery, 2010, 9: 537-550.

11. Liao SQ, Liu YQ, Zeng JY, et al. Aptamer-based sensitive detection of target molecules via RTPCR signal amplication [J]. Bioconjugate Chem, 2010, 21: 2183-2189.

12. Yoshida Y, Horii K, Sakai N, et al. Antibody-specificaptamer-based PCR analysis for sensitive protein detection [J]. Anal Bioanal Chem, 2009, 395: 1089-1096.

13. Constantine NT, Zink H. HIV testing technologies after two decades of evolution [J]. Indian $J$ Med Res, 2005, 121: 519-538.

14. Willner I, Zayats M. Electronic aptamer-based sensors [J]. Angew Chem Int Ed, 2007, 46 (27): 6408-6418. 